\title{
Use of Complementary and Alternative Medicine Among Patients: Classification Criteria Determine Level of Use
}

\author{
Agnete Egilsdatter Kristoffersen, M.A., Vinjar Fønnebø, M.D., M.Sc., Ph.D., \\ and Arne Johan Norheim, M.D., Ph.D.
}

\begin{abstract}
Background and objectives: Self-reported use of complementary and alternative medicine (CAM) among patients varies widely between studies, possibly because the definition of a CAM user is not comparable. This makes it difficult to compare studies. The aim of this study is to present a six-level model for classifying patients' reported exposure to CAM. Prayer, physical exercise, special diets, over-the-counter products/CAM techniques, and personal visits to a CAM practitioner are successively removed from the model in a reductive fashion.

Methods: By applying the model to responses given by Norwegian patients with cancer, we found that $72 \%$ use CAM if the user was defined to include all types of CAM. This proportion was reduced successively to only $11 \%$ in the same patient group when a CAM user was defined as a user visiting a CAM practitioner four or more times. When considering a sample of 10 recently published studies of CAM use among patients with breast cancer, we found 98\% use when the CAM user was defined to include all sorts of CAM. This proportion was reduced successively to only $20 \%$ when a CAM user was defined as a user of a CAM practitioner.

Conclusions: We recommend future surveys of CAM use to report at more than one level and to clarify which intensity level of CAM use the report is based on.
\end{abstract}

\section{Introduction}

$\mathbf{P}$ revalence of self-reported use of complementary and alternative medicine (CAM) among patient groups is difficult to interpret when no standardized way of reporting CAM use has been established. Among patients with cancer, self-reported use of CAM varies between $7 \%{ }^{1}$ and $98 \% .^{2}$ Possible reasons for this wide range in reported use could be differences in the definition of a CAM user ${ }^{3,4}$ and/or differences in timeframe of the use. ${ }^{5}$ Some studies report all possible nonconventional health activities including prayer and exercise as CAM use, ${ }^{6-10}$ others limit it to visits to a CAM therapist or use of CAM techniques, over-the-counter (OTC) products, and dietary changes, ${ }^{5}$ while some limit the reported use of CAM to be only CAM treatment given by a therapist. ${ }^{11,12}$ It is also likely that self-reported use varies dependent on whether the question addresses use of specific CAM methods connected with cancer disease or CAM use in general. These possible methodological differences necessitate a clarification of the criteria used when classifying a person as a CAM user. A more standard series of questions and definitions to generate comparable data has been proposed, ${ }^{3}$ but a comprehensive model for reporting "CAM use in patients" is still lacking.

CAM is mostly defined as something it is not: "A broad set of health care practices that are not part of that country's own tradition and are not integrated into the dominant health care system"; 13 "[a] group of diverse medical and health care systems, practices, and products that are not presently considered to be part of conventional medicine"; 14 and "a diverse group of health-related therapies and disciplines which are not considered to be a part of mainstream medical care."15

Several researchers and institutions have suggested ways of categorizing CAM users. Some classify according to the nature of the treatment, ${ }^{14}$ others classify from the general ac- 
ceptance of the treatment ${ }^{15}$ or from the provider giving the treatment, $^{12}$ and others again from the commitment of use. ${ }^{16}$

Harris and Rees ${ }^{17}$ suggest three categories of CAM use: visiting a practitioner, use of OTC products, and a combination of both. Among the studies included in their review, none supplied information on all of the three categories. Implementation of these three categories is claimed to improve comparability between studies. A subsequent study of CAM use among patients with cancer in Wales with the same first author adds "use of CAM techniques" to the previously suggested categories without a further discussion of a comprehensive model. ${ }^{18}$

The National Center for Complementary and Alternative Medicine (NCCAM) in the United States ${ }^{14}$ classifies CAM practices into four domains: mind-body medicine, biologically based practices, manipulative and body-based practices, and energy medicine. The advantage of the NCCAM model is that a reader will clearly understand which groups of therapies are most commonly used. The major limitation is that the model does not attempt to classify users with regard to overall exposure to CAM treatments. Adding the number of treatments used will not necessarily give a meaningful description of the patient's total exposure to CAM.

The British House of Lords ${ }^{15}$ classifies CAM into three groups: Group 1: professionally organized alternative therapies: acupuncture, chiropractic, herbal, homeopathy, osteopathy. Group 2: complementary therapies: Alexander technique, aromatherapy, Bach and other flower remedies, body work therapies, including massage - counseling stress therapy, hypnotherapy, meditation, reflexology, shiatsu, healing, Maharishi Ayurvedic medicine, nutritional medicine, and yoga. Group 3: alternative disciplines. 3a: long-established and traditional systems of health care: anthroposophic medicine, Ayurvedic medicine, Chinese herbal medicine, Eastern medicine, naturopathy, Traditional Chinese Medicine. 3b: Other alternative disciplines: crystal therapy, dowsing, iridology, kinesiology, radionics. The Select Committee gives this explanation for the three groups: Group 1 includes the most organized professions; Group 2 contains those therapies that most clearly complement conventional medicine, while Group 3 contains therapies that cannot be supported unless convincing research evidence can be produced.

The Ontario Cancer Institute's Guide to Alternative Therapies $^{19}$ suggests classification schemes based on the nature of the CAM intervention and not on the patient using them. Other studies have used questionnaires that include varying CAM treatment modalities without defining overall exposure to CAM in any specific manner. ${ }^{7,20}$

Shmueli and Shuval ${ }^{12}$ suggest that the relationship between conventional and complementary medicine should be taken into account when classifying a patient as a CAM user. They examine three aspects of the relationship: (1) whether only CAM or both conventional and CAM therapies are used for the same problem; (2) whether or not the CAM provider is a medical doctor, and (3) whether or not the referral to the CAM practitioner was made by the attending physician. In their study, they focus on CAM as provided by a CAM practitioner and do not include as "users" persons who used herbs or other products as self-medication without consultation with any practitioner.

Balneaves et al. ${ }^{16}$ suggest taking into account the patient's commitment to CAM use based on (1) the number of thera- pies used, (2) the frequency of use, (3) the effort, and (4) the cost associated with use. They also report prevalence of use in three different categories based on a liberal, a conservative, and a very conservative definition of a CAM user. A liberal definition includes use of alternative medical systems, vitamins and mineral supplements, herbal/plant products, pharmacological/biological supplements, dietary therapies/changes, physical/movement therapies, energy therapies, psychologic/expressive therapies, spiritual therapies, and miscellaneous therapies. The conservative definition excludes complementary therapies that are available within the Canadian health care system or are considered a lifestyle choice. The most conservative definition includes only those therapies that are part of a large alternative health care system with a paradigm distinct from allopathic medicine such as homeopathy, etc.

What still seems to be missing internationally is a simple model that can classify an individual's total exposure level to CAM, and at the same time gives room for cultural differences, making studies comparable across cultures. The aim of this study is therefore to present a six-level model for classifying patients' reported exposure to CAM.

\section{Methods}

\section{Constructing the model}

From a CAM practitioner's point of view, many of the existing classification systems seem illogical. The individual's total exposure to CAM treatment(s) has rarely been addressed, and activities that also would be recommended within conventional medicine can be found included in the CAM use definition.

As a start, we distinguish between patients who have consulted a CAM practitioner (two levels of exposure) from patients who only have practiced self-treatment. This identified levels 1-3 of the model. Since several researchers classify activities as diet, exercise, and prayer as CAM, the model needed to accommodate these activities to be comparable to already published studies. Level 4 (diet) and level 5 (exercise) were therefore added to the model. The model with these five levels was presented at several research conferences in Norway, England, and in the United States and was also discussed with CAM researchers and practitioners as well as medical doctors. Arguments for including prayer in the model and leaving it out were put forward and discussed. Through these discussions, we realized that many researchers consider both prayer and healing as one common spiritual CAM category. From our point of view, these are distinctly different activities. To allow the separation of these two, we added CAM level 6 (prayer) to the model.

\section{Testing the model}

To test the usefulness of the proposed model, we applied it to a recent Norwegian study on CAM use among cancer survivors (unpublished). Data on prayer were not collected in that study, and it is therefore only possible to demonstrate the first five levels of the model. The patients were all longterm survivors of cancer despite an expected 5-year survival less than $60 \%$ at the time of diagnosis. Patient information was extracted from the Norwegian Cancer Registry. A 40item, 4-page multiple-choice postal questionnaire on use of 

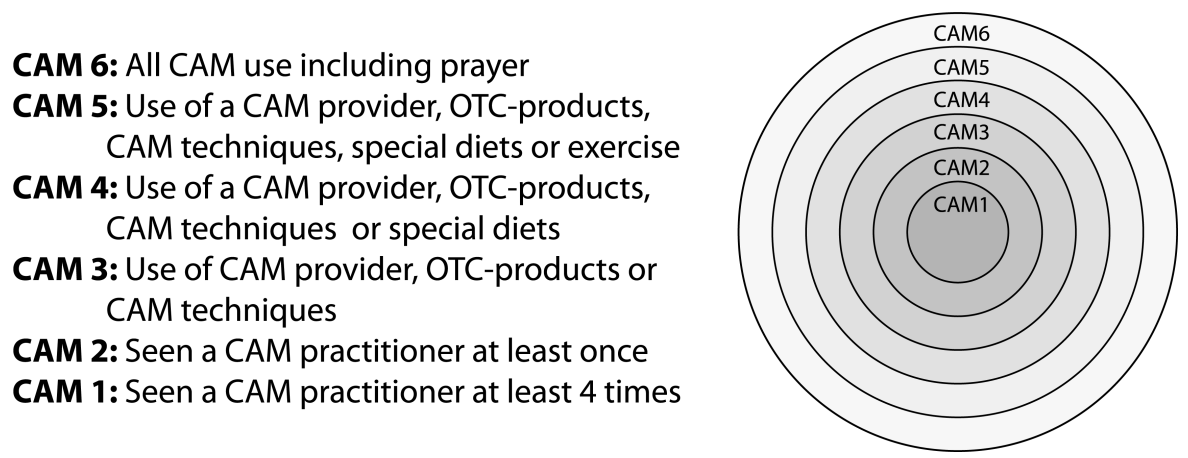

FIG. 1. Accumulative model of complementary and alternative medicine (CAM) level 1-6. OTC, over-the-counter.

CAM was filled in and returned by 400 of 735 included patients (response rate $54 \%$ ) who received the questionnaire, $56 \%$ of the patients were women. Mean patient age was 68 , ranging from 32 to 99 . Mean time from diagnosis was 10.6 years, ranging from 7 to 17 years. The questionnaire included questions about use of CAM providers, use of dietary supplements, practice of self-support, change in diet, and physical activity. It also included sociodemographic variables such as age, educational level, place of residence, and population size of place of residence.

The model was also tested by applying it to recent publications on CAM use among patients with breast cancer. Publications were selected using the MEDLINE ${ }^{\circledR}$ database, with the following search terms: "Breast cancer" in title AND "Complementary Ther" as a MeSH term. This search yielded 121 published studies. We chose the 10 most recent studies with at least 100 patients that describe prevalence of
CAM use after the breast cancer diagnosis was established. Each publication was carefully scrutinized with the purpose of determining at which level CAM use was reported.

\section{Results}

Model

A six-level model describing extent of exposure to CAM use in patients was developed. Six cutoff points were identified that would represent widely accepted levels of exposure to CAM. The model is shown in a generic form in Figure 1.

CAM level 6. When classifying individuals as level 6 CAM users, all reported CAM use is included. Use can include visit(s) to a CAM provider, dietary supplements, self-help CAM techniques, dietary changes, exercise, prayer, or a com-

Table 1. Specific Complementary and Alternative Medicine (CAM) Modalities Included at Every CAM Use Level in the Norwegian Study of CAM Use in Cancer Survivors

CAM user type Measurement in Norwegian study

CAM level $6 \quad$ No data collected

CAM level 5 Persons with at least one treatment encounter in one or more of the following areas: acupuncture, homeopathy, reflexology, healing, natural therapy, massage/aromatherapy, stay at alternative cancer clinic, other kinds of treatment given outside the national health care system, individually adapted herbal cure subscribed by a vitamin and mineral therapist and in addition individuals who have used a self-defined cure made of herbs and vitamin supplements, other supplements and/or participated in self-support groups, done relaxation exercise on their own, done meditation regularly, done positive visualization or partaken in other forms of self-support or changed their diet radically or totally or indicated in the questionnaire that "Physical activity is important to me."

CAM level 4

All CAM users defined at level 5 except individuals who reported no other CAM use than "Physical activity is important to me."

CAM level 3 All CAM users defined at level 4 except individuals who reported no other CAM use than radical changes in their diet.

CAM level 2 Persons with at least one treatment encounter in one or more of the following areas: acupuncture, homeopathy, reflexology, healing, natural therapy, massage/aromatherapy, stay at alternative cancer clinic, other kinds of treatment given outside the national health care system, individually adapted herbal cure prescribed by a vitamin and mineral therapist.

CAM level 1

Persons with at least four treatment encounters in one or more of the areas described at level 2. 
bination of all. This level has been used when defining CAM users in previous studies. ${ }^{7-10,16}$

CAM level 5. Classifying individuals as level 5 CAM users excludes those who only have used prayer as a CAM "treatment." This level otherwise includes all the other possible CAM modalities identified at level 6 .

CAM level 4. Classifying individuals as level 4 CAM users excludes those who only have used exercise or prayer as a CAM "treatment," but retains those with dietary changes, users of OTC products, self-help CAM techniques, and users who have seen a CAM practitioner. This level has been used when defining CAM users in previous studies ${ }^{5,21-24}$

CAM level 3. Classifying individuals as level 3 CAM users limits CAM users to those who have used OTC products or self-help CAM techniques that do not require a personal encounter, as well as all users who have seen a CAM practitioner. This level has been used when defining CAM users in previous studies. ${ }^{25,26}$ Yoga, meditation, visualization, qi gong, and t'ai chi are examples of techniques included in this category.

CAM level 2. Classifying individuals as level 2 CAM users limits CAM users to only those who have visited a CAM practitioner one or more times. Level 2 CAM use is similar to Harris' "CAM use involving a personal encounter with a CAM practitioner"17 and has also been used when defining

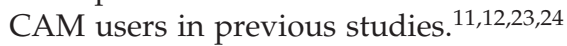

CAM level 1. Finally, classifying individuals as level 1 CAM users requires that the person has seen a CAM practitioner at least four times. Frequent use is suggested as a marker for commitment in use. ${ }^{16}$

All CAM level 1 users are included in levels 2-6, all CAM level 2 users are included in levels 3-6, all CAM level 3 users are included in levels 4-6, all CAM level 4 users are included in levels 5-6 and finally, all CAM level 5 users are included in CAM level 6. Identification of users of the CAM treatment added from levels 1 to 2 can be achieved by subtracting CAM level 1 users from CAM level 2 users. The same approach can be applied if identification of users of added treatment at any level needs to be isolated.

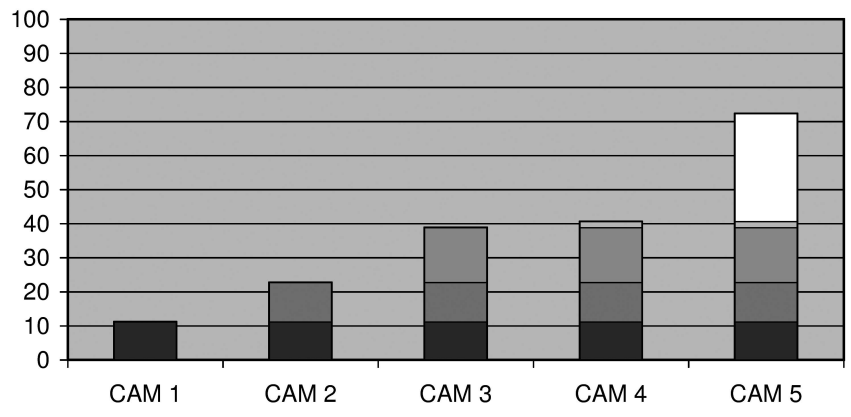

FIG. 2. Self-reported use of complementary and alternative medicine (CAM) dependent on level of CAM use.

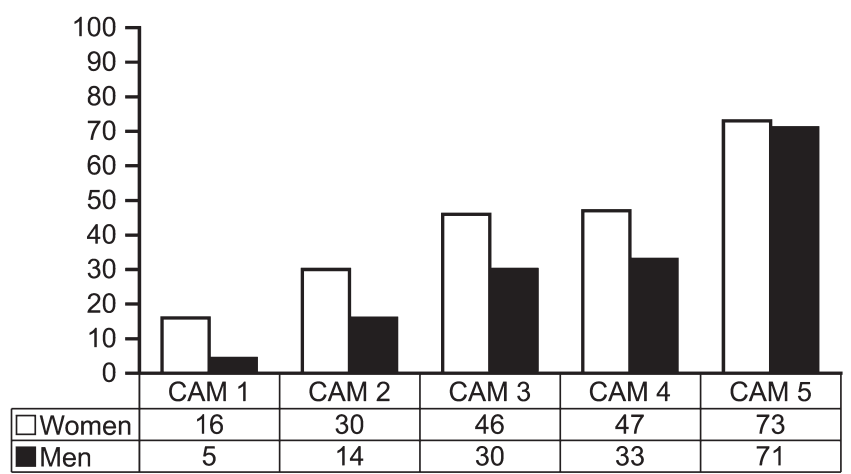

FIG. 3. Self-reported complementary and alternative medicine (CAM) use among men and women dependent on level of use.

The presented model does not specify beforehand which specific treatment modalities to include when establishing each level. This is deliberately done to accommodate cultural differences with regard to what treatments are considered as CAM in each specific environment. Researchers reporting CAM use using this model should, however, specify which practitioners, supplements, techniques, diet programs, physical activity programs, and prayer activity programs they include from their own culture/setting when defining a level.

\section{Variation of Self-Reported CAM Use According to the Six-Level Model}

In the Norwegian study on CAM use among cancer survivors, data on prayer were not collected, and it is therefore only possible to demonstrate the first five levels of the model. By applying the model described above to the responses given by the patients in the Norwegian study (Table 1), we found large differences in reported prevalence of use dependent on which level of reporting that was chosen. The reported use of CAM varied from $11 \%$ at level 1 to $72 \%$ at level 5 (Fig. 2). Among women, the use of CAM varied between $15 \%$ and $76 \%$ while CAM use among men varied from $5 \%$ to $71 \%$ (Fig. 3). The gender differences in use of CAM are highly significant when reported at CAM level 1, where more than three times as many women than men reported CAM use $(p=0.001)$. At level 5 , on the other hand, we did not find statistically significant differences in CAM use between men and women. The ratio here was close to 1 ( $p=$ $0.614)$. In this particular study, the question concerning physical activity was not as stringent as suggested in the model. This might give a higher number of CAM 5 users than what we would have seen if the question was formulated more like that suggested in the model.

Among the recently published studies of CAM use among patients with breast cancer, one study reports CAM use at level 2, ${ }^{27}$ two studies report at level $3,{ }^{28,29}$ six studies report at level $4,,^{30-35}$ and the last study reports at level $5 .^{2}$ The level 2 study reports $20 \%$ use, ${ }^{27}$ the level 3 studies report $33 \%$ and $62 \%{ }^{29}$ use (weighted mean $56 \%$ ), the level 4 studies vary from $20 \%{ }^{30}$ to $69 \%^{35}$ use (weighted mean $47 \%$ ), while the level 5 study reports $98 \%^{2}$ use (Table 2, Fig. 4).

By applying our model to these studies, it was thus possible to partly account for the extremely wide variation in reported CAM use. Residual variation within each level will 


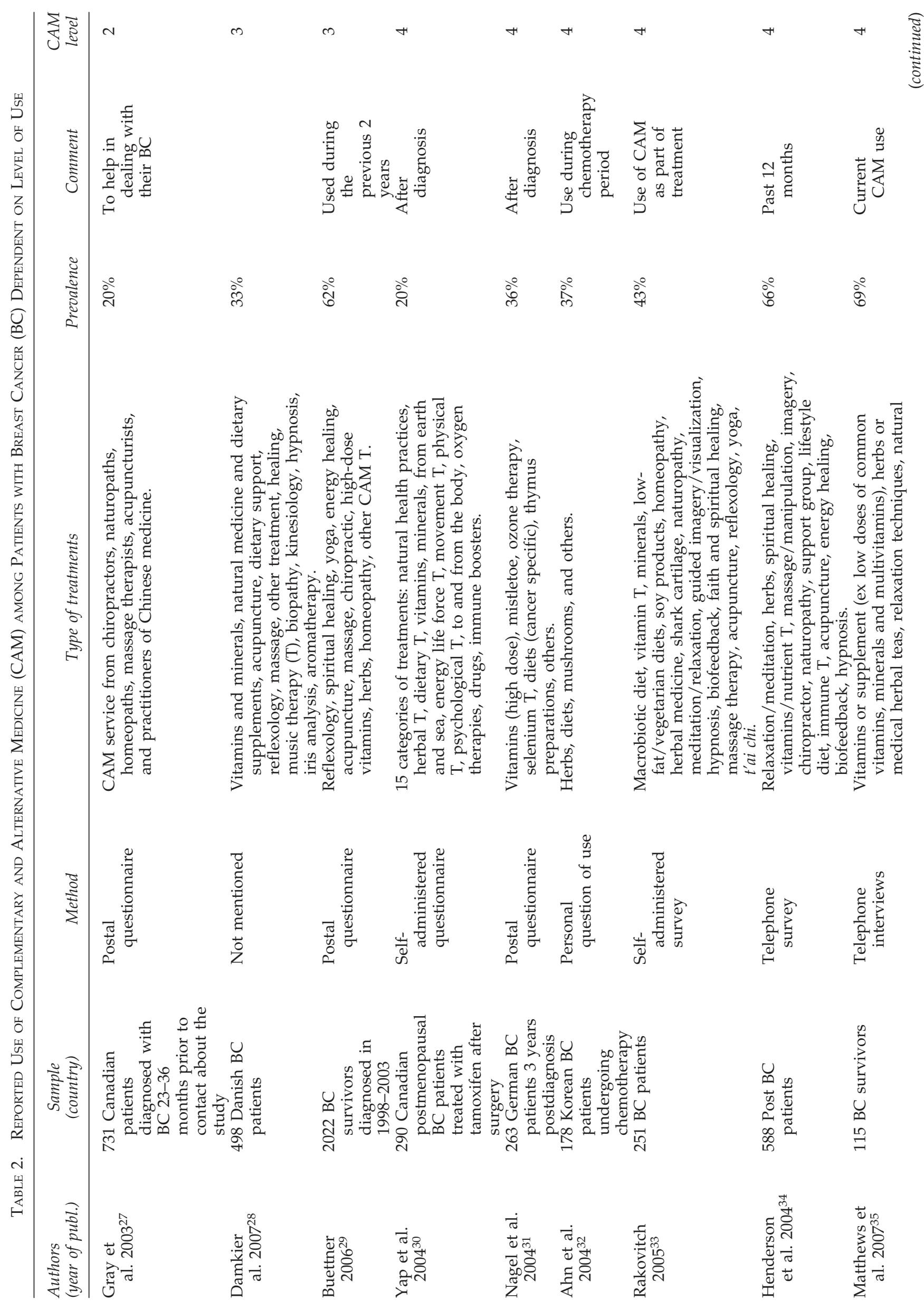




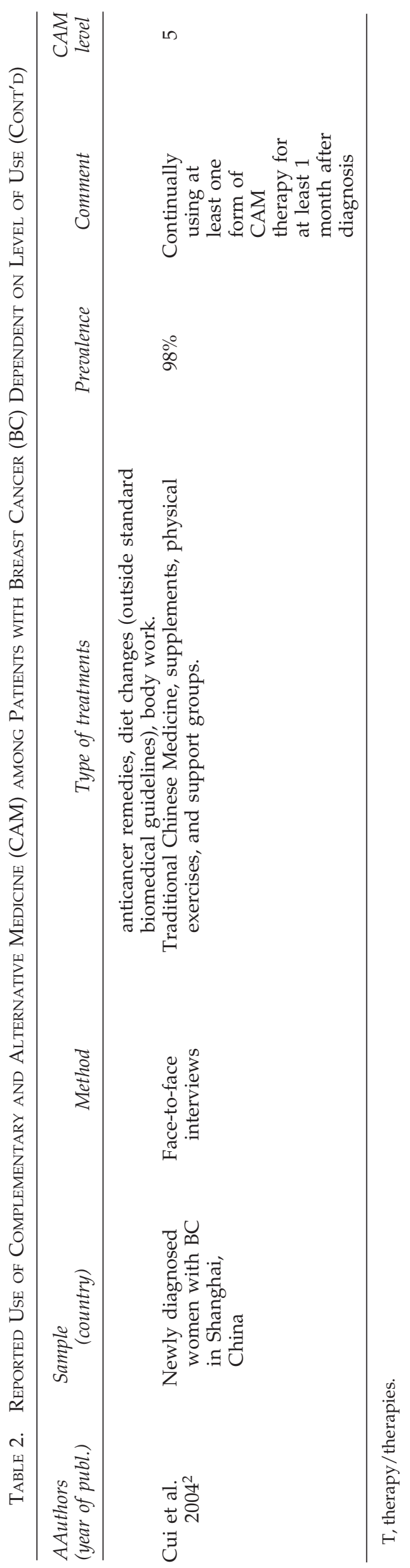




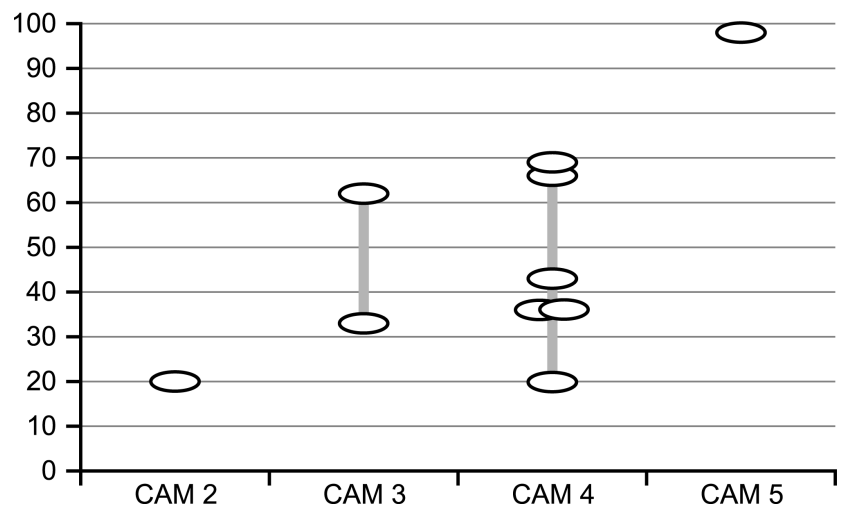

FIG. 4. Complementary and alternative medicine (CAM) use among patients with breast cancer depending on level of use.

of course be expected, given that the studies come from several continents and in addition differ somewhat with regard to the number of specific treatments explored.

\section{Discussion}

Use of a model will always imply limitations. Different countries and cultures have different traditions with regard to complementary and/or alternative medicine. The suggested model can generally accommodate these local variations because the locally used modalities themselves are fairly easy to place in the indicated generic categories of the model.

One major potential limitation is how to relate to traditional folk medicine. Several indigenous populations are reluctant to classify their traditional treatment as CAM. However, in the development of this model we have assumed traditional folk medicine to be a part of broadly defined CAM use, although the World Health Organization distinguishes between the two. ${ }^{13}$

There will, however, be a few treatment modalities that do not readily fit any category, and also some modalities that could possibly fit into more than one category. Treatment support by telephone/mail and the self-practice of CAM techniques with a personal encounter are two examples that might fit into more than one category. We suggest these to be placed in the category that includes a treatment provider (CAM 1-2).

Treatments that are performed by a treatment provider without personal contact, such as distant healing, can also be difficult to classify. In some cases the healer can provide distant healing daily over a long period of time, even though the contact between the patient and the therapist is limited to one telephone call. We suggest this to be classified as treatment including a personal encounter (CAM 1-2) and that the number of treatments is counted as the number of treatments the treatment provider has carried out. Self-treatment based on information in books, the Internet, and friends should be placed in the category not including a treatment provider (CAM 3-6).

The borderline between religious healing and prayer can be vague. Many researchers therefore combine the two in the same question when asking about CAM use. We suggest that religious healing is classified as CAM level 1-2 (seeing a provider) when the provider of this healing is considered a person with special "gifts" and at CAM level 6 when prayer is conducted by the user him/herself or by a priest / church community with no special "gifts."

Some patients increase the amount of healthy food in their diet and exercise more as a result of their current disease without changing to a totally new recommended dietary system. We do not recommend these changes to be considered CAM use.

Other challenges to consider are within what timeframe the use is reported. Some studies report "ever use," others "since diagnosis," "current use," "used within the last 3 or 12 months," or within the last 14 days. One study reports "use that was helpful to their recovery."

We recommend reporting CAM use "Since diagnosis" when a definite diagnosis has been established, and within the last year in other circumstances to optimize the comparability between studies.

\section{Implications of the Model}

CAM level 1 is a category that is not commonly seen in published studies to date. We think it is important to distinguish a comprehensive course of CAM treatment involving personal encounters from just a casual visit or solely selfadministered CAM use. It is important to identify this group because they are the ones choosing an alternative or complementary comprehensive treatment regimen to treat their condition over a potentially important period of time. The patients seeing a provider from one to three times probably represent patients trying a CAM modality without completing a comprehensive series of treatments. The cutoff point of four or more visits is of course partly arbitrary in nature, but does represent a longer treatment trajectory. This cutoff point has also been used in at least one study that has attempted to distinguish at this dimension. ${ }^{36}$

Using CAM level 2 as an identification of CAM users follows international recommendations to delineate CAM users going to CAM providers from a broader definition of CAM use, where use of OTC products could be the only CAM use reported. ${ }^{17}$ The individuals in this category who do not simultaneously fulfill the CAM level 1 criterion might represent those individuals only trying out alternative therapy but not deciding to continue with a full treatment plan. We think the CAM level 3 definition of a CAM user represents what people broadly define as a possible CAM user. CAM levels 4 and 5 can be useful if the focus of a study is to include individuals using dietary change and exercise involvement in the definition of a CAM user. These individuals are thus distinguished from individuals with "normal" attention to diet and "routine" self-care. ${ }^{4}$ These are elements that have been advocated in the conventional health care system for a long time and are considered "lifestyle-oriented" therapies. As seen in the Norwegian study, the proportion of "CAM users" increases substantially when exercise (CAM level 5) is considered CAM. Interestingly, most patients who make major changes in their diet have already utilized CAM at levels 1-3. Identifying CAM users at CAM level 6 is useful in order to be able to compare studies that have included prayer as CAM treatment. ${ }^{7,16}$ 
To report CAM use on various levels as suggested in the model might serve several purposes. A medical doctor needs to be informed about the number of patients receiving CAM treatment to consider whether he or she needs to make an effort to ensure that CAM therapy does not interact negatively with ongoing conventional treatment. We believe that this will be in the interest of the patient. ${ }^{20}$ Use of only OTC products and/or CAM techniques without a provider (the difference between CAM 2 and CAM 3 level use) might require extra awareness from the doctor since there is no CAM provider who is monitoring the treatment. If many patients are CAM users at levels 1-2, the medical doctor and alternative treatment practitioner can ideally enter into a dialogue and, if possible, coordinate and/or integrate their treatment efforts.

Since health authorities have an overall regulatory responsibility, they will need information on the proportion of users who are CAM 1-2 users if they consider integrating provision of CAM treatment with conventional care.

For researchers, the suggested model is useful for a more valid and reliable comparison of prevalence of CAM use between populations and studies. The model can accommodate cultural differences concerning what treatments are considered CAM at different levels. It also presents a meaningful exposure variable in determining effectiveness of CAM use.

We realize that this model has potential for extension and that further discussions around a model for classifying patients as CAM users is required.

\section{Acknowledgments}

This work has been funded by a grant from The Research Council of Norway and with support from the Norwegian Cancer Registry.

\section{References}

1. Eidinger RN, Schapira DV. Cancer patients' insight into their treatment, prognosis, and unconventional therapies. Cancer 1984;53:2736-2740.

2. Cui Y, Shu XO, Gao Y, et al. Use of complementary and alternative medicine by Chinese women with breast cancer. Breast Cancer Res Treat 2004;85:263-270.

3. Ernst E, Cassileth BR. The prevalence of complementary/alternative medicine in cancer: A systematic review. Cancer 1998;83:777-782.

4. Cassileth BR, Vickers AJ. High prevalence of complementary and alternative medicine use among cancer patients: Implications for research and clinical care. J Clin Oncol 2005;23:2590-2592.

5. Risberg T, Lund E, Wist E, et al. Cancer patients use of nonproven therapy: A 5-year follow-up study. J Clin Oncol 1998;16:6-12.

6. Yates JS, Mustian KM, Morrow GR, et al. Prevalence of complementary and alternative medicine use in cancer patients during treatment. Support Care Cancer 2005;13:806-811.

7. Richardson MA, Sanders T, Palmer JL, et al. Complementary/alternative medicine use in a comprehensive cancer center and the implications for oncology. J Clin Oncol 2000;18:2505-2514.

8. Eisenberg DM, Kessler RC, Foster C, et al. Unconventional medicine in the United States: Prevalence, costs, and patterns of use. NEJM 1993;328:246-252.
9. Sparber A, Bauer L, Curt G, et al. Use of complementary medicine by adult patients participating in cancer clinical trials. Oncol Nurs Forum 2000;27:623-630.

10. Hann D, Baker F, Denniston M, et al. Long-term breast cancer survivors' use of complementary therapies: Perceived impact on recovery and prevention of recurrence. Integr Cancer Ther 2005;4:14-20.

11. Sibbritt D, Adams J, Easthope G, et al. Complementary and alternative medicine (CAM) use among elderly Australian women who have cancer. Support Care Cancer 2003;11: 548-550.

12. Shmueli A, Shuval J. Complementary and alternative medicine: Beyond users and nonusers. Complement Ther Med 2006;14:261-267.

13. WHO: General Guidelines for Methodologies on Research and Evaluation of Traditional Medicine. Online document at: www.ordinefoce.it/site/varieda0304/WHO4.doc Accessed October 15, 2008.

14. NCCAM. What Is Complementary and Alternative Medicine (CAM)? NCCAM publication no. D347 February 2007. Online document at: http://nccam.nih.gov/health/ whatiscam/pdf/D347.pdf Accessed October 15, 2008.

15. House of Lords. Complementary and Alternative Medicine: Science and Technology, Sixth Report. Online document at: www.publications.parliament.uk/pa/ld199900/ldselect/ld stech/123/12 Accessed October 15, 2008.

16. Balneaves LG, Bottorff JL, Hislop TG, Herbert C. Levels of commitment: Exploring complementary therapy use by women with breast cancer. J Altern Complement Med 2006; 12:459-466.

17. Harris P, Rees R. The prevalence of complementary and alternative medicine use among the general population: A systematic review of the literature. Complement Ther Med 2000;8:88-96.

18. Harris P, Finlay IG, Cook A, et al. Complementary and alternative medicine use by patients with cancer in Wales: A cross sectional survey. Complement Ther Med 2003;11: 249-253.

19. Tataryn DJ. Paradigms of health and disease: A framework for classifying and understanding complementary and alternative medicine. J Altern Complement Med 2002;8: 877-892.

20. Barnes P, Powell-Griner E, McFann K, Nahin R. CDC Advance Data Report \#343: Complementary and Alternative Medicine Use Among Adults. United States, 2002 May 27, 2004. Online document at: http://nccam.nih.gov/news/ report.pdf Accessed October 15, 2008.

21. Molassiotis A, Scott JA, Kearney N, et al. Complementary and alternative medicine use in breast cancer patients in Europe. Support Care Cancer 2006;14:260-267.

22. Lee MM, Lin SS, Wrench MR, et al. Alternative therapies used by women with breast cancer in four ethnic populations. J Natl Cancer Inst 2000;92:42-47.

23. Boon H, Stewart M, Kennard MA, et al. Use of complementary/alternative medicine by breast cancer survivors in Ontario: Prevalence and perceptions. J Clin Oncol 2000; 18:2515-2521.

24. Boon HS, Olatunde F, Zick SM. Trends in complementary/alternative medicine use by breast cancer survivors: Comparing survey data from 1998 and 2005. BMC Womens Health 2007;7:4.

25. Burstein HJ, Gelber S, Guadagnoli E, et al. Use of alternative medicine by women with early-stage breast cancer. NEJM 1999;340:1733-1739. 
26. Ashikaga T, Bosompra $\mathrm{K}, \mathrm{O}^{\prime}$ Brien $\mathrm{P}$, et al. Use of complementary and alternative medicine by breast cancer patients: Prevalence, patterns and communication with physicians. Support Care Cancer 2002;10:542-548.

27. Gray RE, Fitch M, Goel V, et al. Utilization of complementary/alternative services by women with breast cancer. J Health Soc Policy 2003;16:75-84.

28. Damkier A, Paludan-Muller C, Knoop AS. Use of complementary and alternative medicine (CAM) in breast cancer patients: Efficacy and interactions. Ugeskr Laeger 2007;169: 3111-3114.

29. Buettner C, Kroenke CH, Phillips RS, et al. Correlates of use of different types of complementary and alternative medicine by breast cancer survivors in the nurses' health study. Breast Cancer Res Treat 2006;100:219-227.

30. Yap KP, McCready DR, Fyles A, et al. Use of alternative therapy in postmenopausal breast cancer patients treated with tamoxifen after surgery. Breast J 2004;10:481-486.

31. Nagel G, Hoyer H, Katenkamp D. Use of complementary and alternative medicine by patients with breast cancer: $\mathrm{Ob}$ servations from a health-care survey. Support Care Cancer 2004;12:789-796.

32. Ahn JH, Kim SB, Yun MR, et al. Alternative therapy and abnormal liver function during adjuvant chemotherapy in breast cancer patients. J Korean Med Sci 2004;19:397-400.
33. Rakovitch E, Pignol JP, Chartier C, et al. Complementary and alternative medicine use is associated with an increased perception of breast cancer risk and death. Breast Cancer Res Treat 2005;90:139-148.

34. Henderson JW, Donatelle RJ. Complementary and alternative medicine use by women after completion of allopathic treatment for breast cancer. Altern Ther Health Med 2004;10:52-57.

35. Matthews AK, Sellergren SA, Huo D, et al. Complementary and alternative medicine use among breast cancer survivors. J Altern Complement Med 2007;13:555-562.

36. Tromsø TUo: The Tromsø study. Online document at: http://uit.no/tromsoundersokelsen/forsiden/?Language $=$ en Accessed October 15, 2008.

Address reprint requests to: Agnete Egilsdatter Kristoffersen, M.A. National Research Centre in Complementary and Alternative Medicine

Tromsø Science Park University of Tromsø N-9037 TROMS $\varnothing$ Norway

E-mail: Agnete.Kristoffersen@fagmed.uit.no 
